\title{
Intensive glucose control and macrovascular outcomes in type 2 diabetes. Reply to Emanuele NV [letter] and Yudkin JS, Richter B [letter]
}

\author{
The CONTROL Group
}

Received: 21 September 2009 /Accepted: 22 September 2009/Published online: 12 November 2009

(C) Springer-Verlag 2009

To the Editor: The meta-analysis by the Collaborators on Trials of Lowering Glucose (CONTROL) Group [1] was a collaborative initiative established to address uncertainty about the effects of intensive glucose-lowering on the risk of major cardiovascular events. The four trials included in the meta-analysis were selected on the basis of prespecified trial inclusion criteria. The other important elements of the meta-analysis, including patient subgroups and the analytic strategy, were defined a priori and were not driven by the interim findings of our meta-analysis. As a result we believe we have provided a balanced interpretation of our findings. We agree with Drs Yudkin and Richter [2] that there is much to be gained from further interrogation of the trial data, particularly in relation to the different patient characteristics (e.g. disease history) and to the different drug regimens employed in each trial. We also agree that a meta-analysis that uses individual patient data is optimal for this purpose and allows the full exploration of as much phenotypic information as possible. Such an analysis is planned by the CONTROL Group once all data become available. In the interim, post hoc analyses [3] are not the optimal way of identifying how best to protect diabetic patients from stroke and coronary heart disease.

Duality of interest H. C. Gerstein has received speaker's honoraria and consulting fees from sanofi-aventis, GlaxoSmithKline, Novo Nordisk, Roche and Lilly for topics related to the role of glucose lowering drugs for glucose control and clinical outcomes, and his institution has received grants from sanofi-aventis and GlaxoSmithKline related to clinical trials of glucose-lowering. C. Abrairia has received speaker fees from sanofi-aventis, Takeda and Novo Nordisk. J. Chalmers has received research grants from Servier, administered through the University of Sydney, as chief investigator for ADVANCE. F. Turnbull, J. Chalmers, A. Patel, B. Neal and M. Woodward have received lecture fees from Servier for speaking at scientific meetings.

\section{References}

1. Turnbull FM, Abraira C, Anderson RJ et al (2009) Intensive glucose control and macrovascular outcomes in type 2 diabetes. Diabetologia 52:2288-2298. Erratum 52:2470

2. Yudkin JS, Richter B (2009) Using individual patient data in metaanalyses of glucose-lowering studies. Diabetologia. doi:10.1007/ s00125-009-1564-8

3. Emanuele NV (2009) Duration of diabetes, glucose control and cardiovascular risk. Diabetologia. doi:10.1007/s00125-009-1563-9

The CONTROL Group

The George Institute for International Health,

University of Sydney,

PO Box M201 Missenden Rd,

Sydney NSW 2050, Australia

e-mail: fturnbull@george.org.au 